\title{
Preliminary toxicity results using partial breast 3D-CRT with once daily hypo-fractionation and deep inspiratory breath hold
}

Roman O. Kowalchuk ${ }^{1 *}$ (D, Kara D. Romano ${ }^{1}$, Daniel M. Trifiletti ${ }^{1}$, Sunil W. Dutta ${ }^{1}$, Timothy N. Showalter ${ }^{1}$ and Monica M. Morris ${ }^{1,2}$

\begin{abstract}
Background: To evaluate the clinical outcomes of patients treated with 3D conformal Hypo-fractionated, deep Inspiratory breath-hold (DIBH), Partial breast radiotherapy, termed "HIP." HIP was implemented to merge the schedule of once-daily breast hypofractionation with partial breast treatment.

Methods: We identified 38 breast cancers in 37 patients from 2013 to 2014 treated at our institution with HIP following lumpectomy for early stage breast cancer. Patients received a hypo-fractionated course ( $\leq 20$ fractions) of once daily radiation to the partial breast (lumpectomy cavity + margin) utilizing DIBH regardless of laterality. Clinical and treatment-related characteristics were obtained, including target volume and organ at risk (OAR) dosimetric characteristics. Patients were followed clinically and with at least yearly mammograms for up to 36 months (range 5-36 months). Acute and late toxicity was scored using the Common Terminology Criteria for Adverse Events (CTCAE) v4.03.

Results: Patients received a median dose of 42.56 Gy in 16 Fractions (Fx) (range 40.05-53.2 Gy; and 15-20 Fx). OAR doses were low, with a mean heart dose of $0.37 \mathrm{~Gy}$, an ipsilateral lung V20 mean of $4 \%$, and a contralateral lung V5 of $1 \%$. Acute toxicity ( $\leq$ grade 2$)$ was present in $79 \%(n=30)$ of the cases, with dermatitis being the most common finding (63\%). Late grade $1-2$ toxicity was present in $42 \%(n=16)$ of the cases, with hyperpigmentation being the most common finding $(n=9)$. There were no severe acute or late toxicities ( $\geq$ grade 3 ). At a median follow up of 21 months, there were no local, regional, or distant failures.

Conclusions: We report limited toxicity in this low risk cohort of patients with early stage breast cancer treated with HIP, a unique and logical combination of 3-D conformal external beam radiotherapy, moderate hypo-fractionation, and $\mathrm{DIBH}$.
\end{abstract}

Keywords: Breast cancer, Radiotherapy, Hypo-fractionation, Acute toxicity

\footnotetext{
* Correspondence: rok4nm@virginia.edu

'Department of Radiation Oncology, University of Virginia, 1240 Lee Street,

Box 800383, Charlottesville, VA 22908, USA

Full list of author information is available at the end of the article
}

(C) The Author(s). 2018 Open Access This article is distributed under the terms of the Creative Commons Attribution 4.0 International License (http://creativecommons.org/licenses/by/4.0/), which permits unrestricted use, distribution, and reproduction in any medium, provided you give appropriate credit to the original author(s) and the source, provide a link to the Creative Commons license, and indicate if changes were made. The Creative Commons Public Domain Dedication waiver (http://creativecommons.org/publicdomain/zero/1.0/) applies to the data made available in this article, unless otherwise stated. 


\section{Background}

Breast cancer is the most commonly diagnosed malignancy among women in the United States. In 2013, there were an estimated 232,340 new cases accounting for $29 \%$ of all newly diagnosed cancers [1]. Breast conservation therapy (BCT), which includes lumpectomy (surgical resection of the tumor alone) and radiation therapy (RT), is the nationally accepted standard treatment approach for early stage breast cancer and is used in $70 \%$ of such patients [2, 3].

The use of adjuvant whole breast irradiation (WBI) following breast conservation surgery has been demonstrated in numerous clinical trials to reduce the rates of ipsilateral breast tumor recurrence (IBTR) by over $50 \%$ compared to lumpectomy alone $[4,5]$. The advantage of $\mathrm{BCT}$ over mastectomy is avoidance of a larger surgical procedure, shorter surgical recovery time, superior cosmetic outcomes, and organ preservation; however, the disadvantage of adjuvant WBI is the prolonged treatment time of 3-7 weeks and additional radiation-induced toxicities. With strong clinical evidence to support its use, hypo-fractionation has become increasingly popular for patients in Canada and the United States for early stage breast cancer [2, 6, 7]. There is sufficient evidence from randomized clinical trials that hypo-fractionated RT is now considered the national preferred option for the majority of patients $[6,8,9]$.

Approximately $75 \%$ of breast tumor recurrences occur within or near the lumpectomy cavity, triggering attempts to decrease the volume of breast treated with radiation [10-12]. PBI in conjunction with further hypofractionation, termed accelerated partial breast irradiation (APBI) can be delivered in several forms - brachytherapy with multicatheter balloons, brachytherapy with interstitial implants, or 3-dimensional conformal external beam radiotherapy (3D-CRT). Early data suggests that APBI is safe and effective, and it has been endorsed by the NCCN Guidelines [2]. Various selection criteria exist to guide clinicians in choosing the optimal patients for this technique, but generally, women at sufficient risk for local failure warranting adjuvant therapy, but not regional or distant failure, should be considered [13-16].

The optimal APBI dose and fractionation is currently unclear. The National Surgical Adjuvant Breast and Bowel Project (NSABP) B-39 clinical trial used a dose of $38.5 \mathrm{~Gy}$ in 10 fractions delivered twice daily for 3D-CRT and 34 Gy in 10 fractions for brachytherapy [17]. The 38.5 Gy dose was chosen as the biologically equivalent dose (BED) to 45 Gy in 25 fractions assuming an alpha/ beta ratio of 10 . This same dose was used in the Radiation Therapy Oncology Group (RTOG) 0319 clinical trial [18]. However, this dose and fractionation has led to concerns regarding the increased incidence of late cosmetic toxicity, including subcutaneous fibrosis and fat necrosis [19]. Further, some patients and providers may prefer once-daily fractionation schedules over twice-daily. IMRT has also been used with APBI, notably in the Florence NCT02104895 trial. A dose of 30 Gy in 5 daily fractions was compared to WBI of 50 Gy in 25 fractions, with a boost of $10 \mathrm{~Gy}$ in five fractions. In 520 patients, no significant difference was found in ipsilateral breast tumor recurrence or overall survival, and improved acute and late cosmetic outcomes were found in the APBI group [20]. The use of interstitial multicatheter brachytherapy for APBI has been studied, and Strnad et al. presented five year results of non-inferiority of local recurrence (1.44\%) and side effects [21].

Taken together, there is strong evidence for the use of moderate hypo-fractionation (15-20 fractions) and PBI. A technique for hypo-fractionated PBI using 3D-CRT with deep inspiratory breath hold (HIP: hypo-fractionated inspiratory partial breast irradiation) was implemented at our institution. The 3D-CRT PBI is a particularly useful treatment technique, as it is applicable and readily available to nearly all centers. HIP merged two types of radiation therapy in a novel way by offering "Canadian" hypo-fractionation (15-20 daily fractions) with a partial breast volume. Deep inspiration breath-hold (DIBH) was employed to reduce cardiac dose [22-24] and improve target volume coverage by immobilizing the breast and decreasing toxicity to the ipsilateral lung [25].

This study seeks to identify early clinical outcomes of early stage breast cancer, including efficacy and toxicity associated with HIP.

\section{Methods}

Data collection

We conducted a retrospective analysis of 37 patients with 38 breast cancers treated with3D-CRT, hypo-fractionated PBI from 2013 to 2014 under our uniform institutional policy for HIP during this time period. Approval was obtained from institutional review board (IRB) prior to evaluating outcomes for these patients.

Clinical data was recorded for all eligible patients including: age, stage, histology, grade, estrogen/progesterone receptor status, Her-2-neu amplification status, lympho-vascular space invasion, multifocal disease, surgical margins, menopausal status, and adjuvant therapies (endocrine therapy or chemotherapy).

\section{Treatment planning}

\section{CT simulation and technique}

All patients underwent a computed tomography (CT) simulation positioned on a breast board in the supine position with arms raised overhead and a Vac-Lok (Med Tech Inc., Orange City, IA) for custom immobilization. Radio-dense markers were placed at the time of simulation to delineate the visible borders of breast tissue and the 
lumpectomy scar. DIBH technique was used for all patients regardless of laterality to increase the accuracy of the target volume location and reduce heart dose, as previously reported [26]. Patients were positioned with both arms raised over the head and custom immobilization. Then, the Varian real-time position management (RPM) system was used to initiate DIBH imaging and monitor the duration and displacement of each patient's breath hold. This RPM signal was used as a baseline, and upper and lower limits of $0.5 \mathrm{~cm}$ from the baseline were established, such that treatment would only take place if the patient's breath hold was within this displacement gate. Free breathing was allowed between treatment fields or if multiple breath holds were required for a given field [26]. All patients were treated with PBI using a once-daily fractionation schedule with a 3D conformal plan using multiple non-coplanar beams. Image-guidance included first day MV portal imaging and daily $\mathrm{kV}$ imaging to verify positioning and alignment.

\section{Target volumes}

The partial breast target volumes were based on the RTOG 1005 and NSABP B-39 clinical trials [17, 27]. The lumpectomy gross tumor volume (GTV) included all clinical and radiographic information of the excision cavity, architectural distortion, lumpectomy scar, seroma, and/or surgical clips. The use of surgical clips is standard for such cases at this institution. The lumpectomy clinical target volume (CTV) was defined as GTV + 10$15 \mathrm{~mm}$ uniform expansion and this was limited posteriorly at the anterior surface of the pectoralis muscles and anteriorly $5 \mathrm{~mm}$ from skin. The lumpectomy planning target volume (PTV) was defined as CTV +7 $10 \mathrm{~mm}$. The beam apertures were chosen based on the PTV. To evaluate our target volume coverage and dose-volume constraints, the PTV was copied to create a PTV_Eval. The PTV_Eval was defined as PTV excluding the region outside of breast tissue, i.e. cropped posteriorly at the anterior border of the pectoralis muscles and anteriorly $5 \mathrm{~mm}$ from skin. One characteristic treatment plan is shown in Fig. 1.

\section{Plan evaluation}

Treatment related characteristics and dosimetric data were recorded including: laterality, total dose, number of fractions, and organ at risk data including heart mean dose, volume of heart receiving 20 Gy or more (heart V20), volume of ipsilateral lung receiving $20 \mathrm{~Gy}$ or more (ipsilateral lung V20), volume of contralateral lung receiving $5 \mathrm{~Gy}$ or more (contralateral lung V5), contralateral breast maximum and mean.

\section{Treatment outcomes}

During treatment, patients were evaluated by a physician at least once weekly, including the radiation and breast oncology teams. Clinical follow up data includes history, physical exam, and mammograms. Breast cancer recurrence was recorded as: ipsilateral breast tumor recurrence (IBTR) (in the PTV, outside the PTV, or skin), ipsilateral nodal recurrence, contralateral breast failure, or distant failure.

Toxicity was graded according to the Common Toxicity Criteria for Adverse Events (CTCAE v4.03) to evaluate both acute ( $\leq 90$ days post-treatment) and late (>90 days post-treatment) findings [28]. Toxicities were reported as fatigue, breast pain, dermatitis, pruritus, skin hyper/hypo-pigmentation, skin induration, skin ulceration, telangiectasia, breast fibrosis, lung complications, heart complications, and lymphopenia.

\section{Results}

\section{Clinical characteristics}

Of the 38 breast cancers in this study, $23.7 \%$ were stage $0,86.8 \%$ were stage IA, $0 \%$ were stage IB, and $7.9 \%$ were stage IIA. Patient and tumor characteristics are listed in

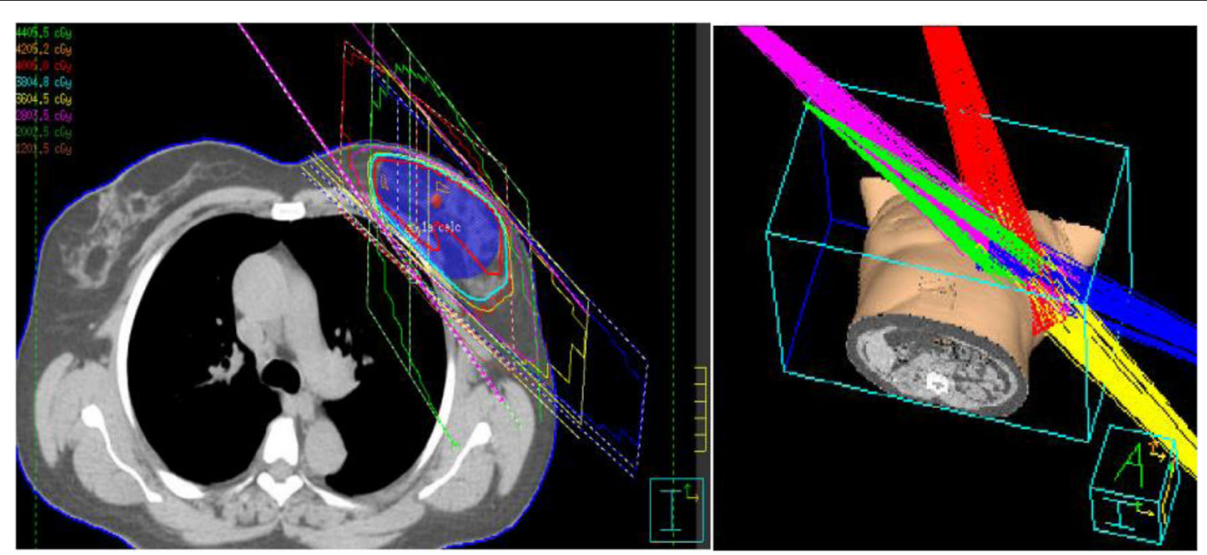

Fig. 1 Here is an example of an HIP plan utilizing 5 non-coplanar 3D beams to create a conformal dose distribution to the partial breast to a total dose of 40.05 Gy in 15 Fx 
Table 1 Patient and tumor characteristics of 38 breast cancers and 37 patients

\begin{tabular}{lll}
\hline Characteristics & $\mathrm{n}$ & $\%$ \\
\hline Female & 38 & 100 \\
Age (median, years) & & $\begin{array}{l}62 \text { years } \\
\text { (range: 52-79) }\end{array}$
\end{tabular}

Laterality

Right

Left

Menopausal status

Pre-menopausal

Post-menopausal

Unknown

Surgical margin

Negative

Positive

Surgery, breast

Lumpectomy

Mastectomy

Surgery, Axilla

None

SLNBX

ALND

Histology

DCIS

Invasive Ductal Carcinoma

Invasive Lobular Carcinoma

T stage

Tis

T1a

T1b

T1C

$\mathrm{T} 2$

$\mathrm{N}$ stage

NO

M stage

Mo

LVSI

No

Yes

Not reported

Grade

1

2

3

ER+

Unknown
18

20

\section{7}

53

0

37

1

37

38

0

11

27

0

12

21

5
Table 1 Patient and tumor characteristics of 38 breast cancers and 37 patients (Continued)

\begin{tabular}{lll}
\hline Characteristics & $\mathrm{n}$ & $\%$ \\
\hline PR+ & 20 & 53 \\
Unknown & 8 & 21 \\
HER2+ & 2 & 5 \\
Unknown & 12 & 32 \\
Multi-focal & 14 & 37 \\
Hormonal therapy & 28 & 74 \\
Chemotherapy & 5 & 13
\end{tabular}

Abbreviations: SLNBX sentinel lymph node biopsy, $A L N D$ axillary lymph node dissection, DCIS ductal carcinoma in situ, LVSI lympho-vascular space invasion, $E R$ estrogen receptor, $P R$ progesterone receptor, HER2 human epidermal growth factor receptor 2

Table 1. All patients were deemed "suitable" or "cautionary" per ASTRO consensus guidelines for APBI [16]. While $78.9 \%$ of the patients were considered cautionary per the 2009 recommendations, this number decreased to $36.8 \%$ in the updated 2016 recommendations, as seen in Table 2 [29]. The recent update stated that ductal carcinoma in situ (DCIS) is suitable for APBI if it is screening-detected, low to intermediate nuclear grade, size $\leq 2.5 \mathrm{~cm}$, and resected with margins negative at $\geq 3 \mathrm{~mm}$. Of the cautionary patients, $43 \%$ were estrogen receptor negative, and $36 \%$ had invasive lobular carcinoma (ILC) on histologic review. Clinical outcomes using APBI with ILC have been found to be no different than those with invasive ductal histology, hence their inclusion in this study [30]. There were no unsuitable patients treated in this study.

\section{Treatment characteristics}

The median total dose was 42.56 Gy (range of 40.0553.20 Gy), over a median 16 Fx (range of 15-20 Fx). Table 3 provides radiation treatment characteristics of the entire cohort.

\section{Toxicity}

Toxicity data is listed in Table 4. Acute toxicity of any kind was recorded in 30 of 38 cases (79\%) within 90 days of completion of treatment. There were no grade 3 or

Table 2 Patient category according to ASTRO consensus guidelines for APBI

\begin{tabular}{llll}
\hline ASTRO consensus & Category & $\mathrm{n}$ & $\%$ \\
\hline APBI Group (2009 Original) & Suitable & 8 & 21.1 \\
& Cautionary & 30 & 78.9 \\
& Unsuitable & 0 & \\
APBI Group (2016 Update) & Suitable & 24 & 63.2 \\
& Cautionary & 14 & 36.8 \\
& Unsuitable & 0 & \\
\hline
\end{tabular}

Abbreviations: ASTRO American Society for Radiation Oncology 
Table 3 Radiation treatment characteristics of 38 breast cancers treated with HIP

\begin{tabular}{|c|c|}
\hline Characteristics & Value \\
\hline Total Dose (mean, Gy) & $\begin{array}{l}43.54 \\
\text { (range: 40.05-53.20) }\end{array}$ \\
\hline Total Dose (median, Gy) & 42.56 \\
\hline Total Fractions (mean, Fx) & $\begin{array}{l}16 \\
\text { (range: 15-20) }\end{array}$ \\
\hline Total Fractions (median, Fx) & 16 \\
\hline \multicolumn{2}{|l|}{ Modality } \\
\hline Photon only & $33(86.8 \%)$ \\
\hline Mixed beam (photon/electron) & $5(13.2 \%)$ \\
\hline Heart mean dose (median, Gy) & $\begin{array}{l}0.37 \\
\text { (range: 0.14-0.94) }\end{array}$ \\
\hline Heart mean dose, right breast (mean, Gy) & 0.36 \\
\hline Heart mean dose, left breast (mean, Gy) & 0.38 \\
\hline Heart V20 (mean, \%) & 0 \\
\hline Heart V20 (median, \%) & 0 \\
\hline Ipsilateral lung V20 (median, \%) & 4.0 \\
\hline Contralateral lung V5 (median, $\%, n=17$ ) & 1.3 \\
\hline Contralateral breast Dmax (median, Gy, $n=13$ ) & 1.4 \\
\hline Contralateral breast mean (median, Gy, $n=13$ ) & 0.2 \\
\hline
\end{tabular}

Abbreviations: Dmax maximum point dose

greater acute toxicities. Overall, acute dermatitis (58\% grade 1 and 5\% grade2) was the most common finding, followed by fatigue ( $8 \%$ grade 1 and $5 \%$ grade 2 ) and hyperpigmentation (13\% grade 1$)$. There were no cases of hypopigmentation, ulceration, telangiectasia, or fibrosis.

Late toxicity of any kind was recorded in 16 of 36 cases $(42 \%)$. Long term data was not available for 2 cases. There were no grade 2 or greater late toxicities. Only grade 1 toxicity was noted, and it was most commonly hyperpigmentation (24\%), followed by fibrosis (21\%). There were no cases of late dermatitis, pruritus, hypopigmentation, ulceration, telangiectasia, or pain.

\section{Disease control}

At a median follow up of 21 months, there were no local, regional, or distant failures, including IBTR in and out of the field, skin recurrence, and contralateral breast failure.

\section{Discussion}

Our results demonstrate a favorable rate of acute and late toxicity following HIP. The Whelan Trial reported comparable rates of good or excellent cosmetic outcomes in the control WBI group compared to the hypo-fractionated arm ( $71 \%$ vs. $70 \%)$ at 10 years of follow up [8]. The Royal Marsden Hospital/Gloucestershire Oncology Center (RMH/GOC) trial similarly found that $30.3-45.7 \%$ of patients recorded some change in breast
Table 4 Toxicity following 3D-CRT external beam HIP (according to CTCAE $\vee$ 4.0)

\begin{tabular}{|c|c|c|c|}
\hline & Grade 1 & Grade 2 & Grade 3-5 \\
\hline \multicolumn{4}{|c|}{ Acute Toxicity ${ }^{\mathrm{a}}(n=30$ cases) } \\
\hline Dermatitis & 22 & 2 & 0 \\
\hline Pruritus & 1 & 0 & 0 \\
\hline Hyperpigmentation & 5 & 0 & 0 \\
\hline Hypopigmentation & 0 & 0 & 0 \\
\hline Induration & 2 & 0 & 0 \\
\hline Ulceration & 0 & 0 & 0 \\
\hline Telangiectasia & 0 & 0 & 0 \\
\hline Fibrosis & 0 & 0 & 0 \\
\hline Fatigue & 3 & 2 & 0 \\
\hline Pain & 4 & 1 & 0 \\
\hline \multicolumn{4}{|c|}{ Late Toxicity ${ }^{\mathrm{b}}$ ( $n=16$ cases) } \\
\hline Dermatitis & 0 & 0 & 0 \\
\hline Pruritus & 0 & 0 & 0 \\
\hline Hyperpigmentation & 0 & 0 & 0 \\
\hline Hypopigmentation & 9 & 0 & 0 \\
\hline Induration & 1 & 0 & 0 \\
\hline Ulceration & 0 & 0 & 0 \\
\hline Telangiectasia & 0 & 0 & 0 \\
\hline Fibrosis & 8 & 0 & 0 \\
\hline Fatigue & 1 & 0 & 0 \\
\hline Pain & 0 & 0 & 0 \\
\hline
\end{tabular}

$a_{\leq} \leq 90$ days from the start of radiation therapy

${ }^{b}>90$ days from the start of radiation therapy

appearance at 5 years [31]. Similarly, our results showed that 10 of 38 treatments resulted in breast appearance changes (26.3\%). Further, our data showed no cases of telangiectasia, whereas the $\mathrm{RMH} / \mathrm{GOC}$ trial showed rates of $13.8-14.3 \%$.

Some concerns remain regarding late toxicity and poor cosmesis. The RAPID trial compared standard WBI to $38.5 \mathrm{~Gy}$ in $10 \mathrm{Fx}$ external beam APBI, showing worse grade $1-2$ toxicity and cosmesis in the experimental APBI arm at 3 years [32]. Conversely, the UK IMPORT LOW trial utilized a dose of $40 \mathrm{~Gy}$ in $15 \mathrm{Fx}$ to the partial breast, and this trial not only found PBI at this dose to be non-inferior regarding local relapse but also adverse effects were similar to better when compared to WBI [33]. The PBI group had better late outcomes at 5 years regarding skin change, overall breast appearance, smaller breast, and breast firmness. Thus, although some data raises concern regarding APBI toxicity (e.g. the RAPID trial), there is a growing body of literature demonstrating non-inferior - or even superior - toxicity outcomes [33].

The low incidence of late adverse effects reported in our study coupled with the improved cosmesis in the IMPORT LOW study may reflect the inherent 
differences of dose fractionation. Late-responding normal tissues (e.g. dermal skin) are characterized by a low $\alpha / \beta$ ratio. Figure 2 demonstrates that the frequently utilized dose fractionation schedules for PBI (including 38.5 Gy in $10 \mathrm{Fx}$ ) have similar EQD2 and BED10 values compared to standard WBI hypo-fractionation doses (used in both these patients and the IMPORT LOW study); however, the BED3 and BED 1.5 of normal tissues is substantially higher with the 38.5 Gy in $10 \mathrm{Fx}$ schedule.

DIBH was developed to reduce cardiac dose. Epidemiological studies have found higher mortality from myocardial infarction with left-sided breast RT compared to right-sided, and reduction of cardiac dose has been shown to decrease ischemic heart disease [2224]. In this study, heart V20 was $0 \%$, and the mean heart dose was 0.37 Gy (range 0.14-0.94 Gy). Compared to brachytherapy APBI, DIBH WBI has been shown to have a lower heart dose [34]. In our study, the low heart dose is likely a combination of PBI and DIBH. DIBH also further immobilizes the breast during treatment, improving the accuracy of dose delivery and reducing radiation doses to the ipsilateral lung [35]. In this case, ipsilateral lung V20 had a mean of 4.0\% (range $0-13$ ).

The limitations of this study include the retrospective analysis by nature, which obfuscates detailed cosmetic analysis at long term intervals. The follow up of 22 months

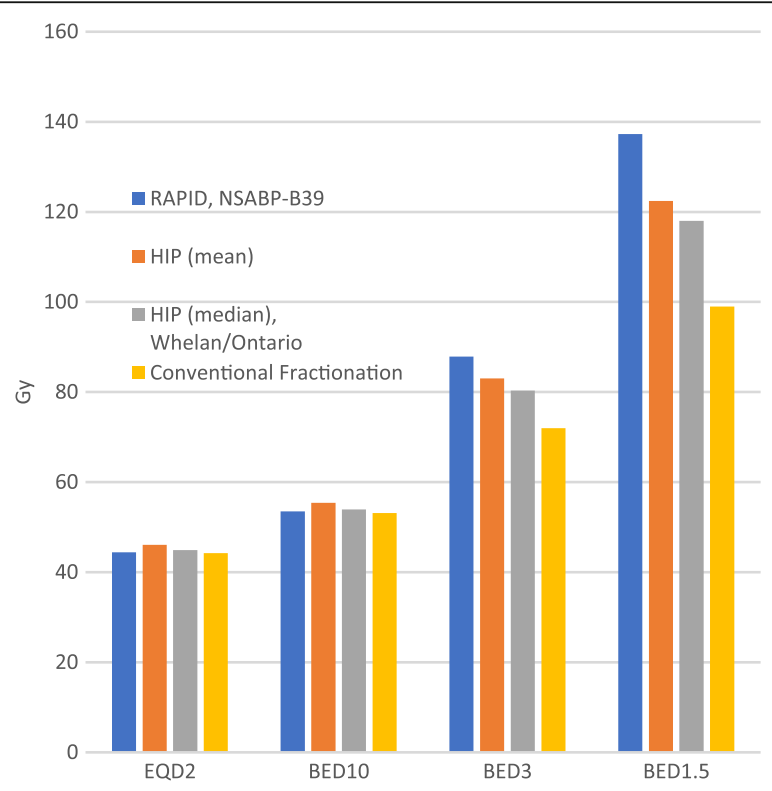

Fig. 2 Dose comparisons of various dose/fractionation schedules for breast irradiation. Blue: 38.5 Gy in 10 Fx (RAPID and NSABP B-39 trials); Red: 43.54 Gy in 16 Fx(mean HIP dose); Green: 42.56 Gy in 16 Fx (median HIP dose, Whelan/Ontario hypo-fractionation); Purple: 45 Gy in 25 Fx (conventional fractionation). Abbreviations: EQD2: equivalent dose in $2 \mathrm{~Gy}$ fractions, $\mathrm{BED}_{10}$ : biologically effective dose for tissue/tumor with an $\alpha / \beta$ ratio of 10,3 and 1.5 respectively is likely insufficient time to capture many instances of IBTR, but it is sufficient to note acute toxicity and most late toxicity associated with RT. The cohort size is small, largely due to the unique scheduling and delivery method applied to this patient set.

\section{Conclusion}

We report limited toxicity without early recurrences in this low risk cohort of patients with early stage breast cancer treated with HIP, a unique combination of 3D-CRT external beam radiotherapy, moderate hypo-fractionation, and DIBH. HIP offers early recurrence and toxicity findings consistent with previous PBI trials, such as IMPORT LOW, and the addition of DIBH offers the potential to further reduce patient motion and toxicity.

\section{Abbreviations}

ALND: Axillary lymph node dissection; APBI: Accelerated partial breast irradiation; ASTRO: American Society for Radiation Oncology; BCT: Breast conservation therapy; $\mathrm{BED}$ : Biologically equivalent dose; $\mathrm{BED}_{10}$ : Biologically effective dose for tissue/tumor with an $\alpha / \beta$ ratio of 10, 3 and 1.5, respectively; CT: Computed tomography; CTCAE v4.03: Common Toxicity Criteria for Adverse Events; CTV: Clinical target volume; DCIS: Ductal carcinoma in situ; DIBH: Deep inspiratory breath-hold; Dmax: Maximum point dose; EQD2: Equivalent dose in 2 Gy fractions; ER: Estrogen receptor; Fx: Fractions; GTV: Gross tumor volume; Gy: Gray; HER2: Human epidermal growth factor receptor 2; HIP: Hypo-fractionated, deep Inspiratory breathhold, Partial breast radiotherapy; IBTR: Ipsilateral breast tumor recurrence; IRB: Institutional review board; LVSI: Lympho-vascular space invasion; NSABP: National Surgical Adjuvant Breast and Bowel Project; PBI: Partial breast irradiation; PR: Progesterone receptor; PTV: Planning target volume; RMH/GOC: Royal Marsden Hospital / Gloucestershire Oncology Center;

RT: Radiation therapy; RTOG: Radiation Therapy Oncology Group; SLNBx: Sentinel lymph node biopsy; V20: Volume of an organ receiving at least $20 \mathrm{~Gy}$; V5: Volume of an organ receiving at least 5 Gy; WBI: Whole breast irradiation

\section{Funding}

This research required no funding.

\section{Availability of data and materials}

The datasets used and/or analyzed during the current study are available from the corresponding author on reasonable request.

\section{Authors' contributions}

ROK was involved in data analysis, writing and drafting the manuscript, and finalizing edits for the manuscript. KDR was also involved in data analysis, writing and drafting the manuscript, and finalizing edits for the manuscript. DMT was primarily involved in analyzing the data and finalizing edits for the manuscript. SWD assisted with editing and finalizing the manuscript. TNS helped with editing and finalizing the manuscript. MMM was involved in primary data collection and finalizing the manuscript. All authors read and approved the final manuscript.

Ethics approval and consent to participate

Approval was obtained from institutional review board (IRB) prior to evaluating outcomes for these patients.

\section{Consent for publication}

Not applicable.

\section{Competing interests}

The authors declare that they have no competing interests. 


\section{Publisher's Note}

Springer Nature remains neutral with regard to jurisdictional claims in published maps and institutional affiliations.

\section{Author details}

'Department of Radiation Oncology, University of Virginia, 1240 Lee Street, Box 800383, Charlottesville, VA 22908, USA. ${ }^{2}$ Department of Radiation Oncology, Virginia Commonwealth University, Richmond, VA, USA.

Received: 6 February 2018 Accepted: 17 July 2018

Published online: 27 July 2018

\section{References}

1. Siegel RL, Miller KD, Jemal A. Cancer statistics, 2017. CA Cancer J Clin. 2017; 67:7-30.

2. Gradishar WJ, Anderson BO, Balassanian R, Blair SL, Burstein HJ, Cyr A, et al. NCCN guidelines insights breast Cancer, version 1.2016. J Natl Compr Cancer Netw. 2015;13:1475-85.

3. Agarwal S, Pappas L, Neumayer L, Kokeny K, Agarwal J. Effect of breast conservation therapy vs mastectomy on disease-specific survival for earlystage breast cancer. JAMA Surg. 2014;149:267-74.

4. Fisher B, Anderson S, Bryant J, Margolese RG, Deutsch M, Fisher ER, et al. Twenty-year follow-up of a randomized trial comparing total mastectomy, lumpectomy, and lumpectomy plus irradiation for the treatment of invasive breast cancer. N Engl J Med. 2002;347:1233-41.

5. Veronesi U, Saccozzi R, Del Vecchio M, Banfi A, Clemente C, De Lena M, et al. Comparing radical mastectomy with quadrantectomy, axillary dissection, and radiotherapy in patients with small cancers of the breast. N Engl J Med. 1981;305:6-11.

6. Smith BD, Bentzen SM, Correa CR, Hahn CA, Hardenbergh PH, Ibbott GS, et al. Fractionation for whole breast irradiation: an American Society for Radiation Oncology (ASTRO) evidence-based guideline. Int J Radiat Oncol Biol Phys. 2011;81:59-68.

7. Tran K, Rahal R, Brundage M, Fung S, Louzado C, Milosevic M, et al. Use of low-value radiotherapy practices in Canada: an analysis of provincial cancer registry data. Curr Oncol. 2016;23:351-5.

8. Whelan TJ, Pignol JP, Levine MN, Julian JA, MacKenzie R, Parpia S, et al. Long-term results of hypofractionated radiation therapy for breast cancer. $N$ Engl J Med. 2010;362:513-20.

9. Kim KS, et al. Hypofractionated whole breast irradiation: new standard in early breast cancer after breast-conserving surgery. Radiat Oncol J. 2016; 34(2):81.

10. Fisher ER, Anderson S, Tan-Chiu E, Fisher B, Eaton L, Wolmark N. Fifteen-year prognostic discriminants for invasive breast carcinoma: National Surgical Adjuvant Breast and bowel project Protocol-06. Cancer. 2001;91:1679-87.

11. Faverly DR, Burgers $L$, Bult $P$, Holland R. Three dimensional imaging of mammary ductal carcinoma in situ: clinical implications. Semin Diagn Pathol. 1994:11:193-8.

12. Kurtz JM, Amalric R, Brandone H, Ayme Y, Spitalier JM. Results of wide excision for mammary recurrence after breast-conserving therapy. Cancer. 1988;61:1969-72.

13. Shah C, Badiyan S, Ben Wilkinson J, Vicini F, Beitsch P, Keisch M, et al. Treatment efficacy with accelerated partial breast irradiation (APBI): final analysis of the American Society of Breast Surgeons MammoSite((R)) breast brachytherapy registry trial. Ann Surg Oncol. 2013;20:3279-85.

14. Shah C, Vicini F, Wazer DE, Arthur D, Patel RR. The American brachytherapy society consensus statement for accelerated partial breast irradiation. Brachytherapy. 2013;12:267-77.

15. Polgar C, Van Limbergen E, Potter R, Kovacs G, Polo A, Lyczek J, et al. Patient selection for accelerated partial-breast irradiation (APBI) after breastconserving surgery: recommendations of the Groupe Europeen de Curietherapie-European Society for Therapeutic Radiology and Oncology (GEC-ESTRO) breast cancer working group based on clinical evidence (2009). Radiother Oncol. 2010;94:264-73.

16. Smith BD, Arthur DW, Buchholz TA, Haffty BG, Hahn CA, Hardenbergh PH, et al. Accelerated partial breast irradiation consensus statement from the American Society for Radiation Oncology (ASTRO). Int J Radiat Oncol Biol Phys. 2009;74:987-1001.

17. Vicini FA, White J, Arthur D. NSABP B-39/RTOG 0413 protocol, A randomized phase III study of conventional whole breast irradiation (WBI) versus partia breast irradiation (PBI) for women with stage 0, I or || breast cancer. 2005.
18. Vicini FA, et al. Initial efficacy results of RTOG 0319: Three dimensional conformal radiation therapy (3D-CRT) confined to the region of the lumpectomy cavity for Stage I//I breast carcinoma. Int J Radiat Oncol Biol Phys. 2008;72(1):S3.

19. Leonard KL, Hepel JT, Hiatt JR, Dipetrillo TA, Price LL, Wazer DE. The effect of dose-volume parameters and interfraction interval on cosmetic outcome and toxicity after 3-dimensional conformal accelerated partial breast irradiation. Int J Radiat Oncol Biol Phys. 2013;85:623-9.

20. Livi $L$, et al. Accelerated partial breast irradiation using intensity-modulated radiotherapy versus whole breast irradiation: 5 -year survival analysis of a phase 3 randomised controlled trial. Eur J Cancer. 2015;51(4):451-63.

21. Strnad V, et al. 5-year results of accelerated partial breast irradiation using sole interstitial multicatheter brachytherapy versus whole-breast irradiation with boost after breast-conserving surgery for low-risk invasive and in-situ carcinoma of the female breast: a randomised, phase 3, non-inferiority trial. Lancet. 2016;387(10015):229-38.

22. Rutter $C E$, Chagpar $A B$, Evans SB. Breast cancer laterality does not influence survival in a large modern cohort: implications for radiation-related cardiac mortality. Int J Radiat Oncol Biol Phys. 2014;90:329-34.

23. Rutqvist $L E$, Johansson $\mathrm{H}$. Mortality by laterality of the primary tumour among 55,000 breast cancer patients from the Swedish Cancer Registry. Br J Cancer. 1990:61:866-8.

24. Latty $D$, et al. Review of deep inspiration breath-hold techniques for the treatment of breast cancer. J Med Radiat Sci. 2015;62(1):74-81.

25. Shim J-G, et al. Dose-volume analysis of lung and heart according to respiration in breast cancer patients treated with breast conserving surgery. J Breast Cancer. 2012;15(1):105-10.

26. Reardon KA, Read PW, Morris MM, Reardon MA, Geesey C, Wijesooriya K. A comparative analysis of 3D conformal deep inspiratory-breath hold and free-breathing intensity-modulated radiation therapy for left-sided breast cancer. Med Dosim. 2013;38:190-5.

27. Radiation Therapy Oncology Group. RTOG 1005: a phase III trial of accelerated whole breast irradiation with hypofractionation plus concurrent boost versus standard whole breast irradiation plus sequential boost for early-stage breast cancer. 2013.

28. US Department of Health and Human Services. Common terminology criteria for adverse events (CTCAE) version 4.0. National Institutes of Health, National Cancer Institute 4.03; 2009.

29. Correa C, Harris EE, Leonardi MC, Smith BD, Taghian AG, Thompson AM, et al. Accelerated partial breast irradiation: executive summary for the update of an ASTRO evidence-based consensus statement. Pract Radiat Oncol. 2017;7:73-9.

30. Shah C, et al. Clinical outcomes using accelerated partial breast irradiation in patients with invasive lobular carcinoma. Int J Radiat Oncol Biol Phys. 2011;81(4)::547-51.

31. Yarnold J, Ashton A, Bliss J, Homewood J, Harper C, Hanson J, et al. Fractionation sensitivity and dose response of late adverse effects in the breast after radiotherapy for early breast cancer: long-term results of a randomised trial. Radiother Oncol. 2005;75:9-17.

32. Olivotto IA, Whelan TJ, Parpia S, Kim DH, Berrang T, Truong PT, et al. Interim cosmetic and toxicity results from RAPID: a randomized trial of accelerated partial breast irradiation using three-dimensional conformal external beam radiation therapy. J Clin Oncol. 2013;31:4038-45.

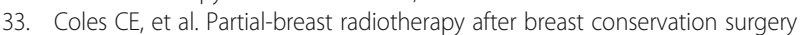
for patients with early breast cancer (UK IMPORT LOW trial): 5 -year results from a multicentre, randomised, controlled, phase 3, non-inferiority trial. Lancet. 2017;390(10099):1048-60.

34. Holliday EB, Kirsner SM, Thames HD, Mason BE, Nelson CL, Bloom ES. Lower mean heart dose with deep inspiration breath hold-whole breast irradiation compared with brachytherapy-based accelerated partial breast irradiation for women with left-sided tumors. Pract Radiat Oncol. 2017;7:80-5.

35. Essers M, Osman SO, Hol S, Donkers T, Poortmans PM. Accelerated partial breast irradiation (APBI): are breath-hold and volumetric radiation therapy techniques useful? Acta Oncol. 2014;53:788-94. 